\title{
Pyrroloquinoline quinone induces chondrosarcoma cell apoptosis by increasing intracellular reactive oxygen species
}

\author{
LINCHUN WEN ${ }^{1}$, XIYAN LU $^{1}$, RUI WANG ${ }^{1}$, XIAOWEI JIN ${ }^{1}$, LIQIANG HU ${ }^{2}$ and CHUANWEN YOU ${ }^{1}$ \\ ${ }^{1}$ Department of Oncology, Suqian People's Hospital of Nanjing Drum Tower Hospital Group, Suqian, \\ Jiangsu 223800; ${ }^{2}$ Department of Hepatobiliary and Pancreatic Surgery, The Second Affiliated Hospital, \\ School of Medicine, Zhejiang University, Hangzhou, Zhejiang 310009, P.R. China
}

Received May 9, 2017; Accepted December 6, 2017

DOI: $10.3892 / \mathrm{mmr} .2018 .8745$

\begin{abstract}
Pyrroloquinoline quinone (PQQ) has been reported to contribute to cancer cell apoptosis and death; however, little is known of its underlying mechanisms. The present study was designed to investigate the role of PQQ in chondrosarcoma cell apoptosis and the underlying mechanism. A cell cytotoxicity assay was used to detect cell death; flow cytometry analysis was also performed to determine cell apoptosis and intracellular reactive oxygen species (ROS). Biochemical methods were employed to detect the activity and the expression of superoxide dismutase (SOD)1, SOD2 and glutathione. The present study also examined the effect on tumorigenesis in vivo. The results demonstrated that the apoptosis of SW1353 cells induced by PQQ increased in a concentration- and time-dependent manner, which may be attributable to the accumulation of intracellular ROS. In the in vivo experiments, PQQ inhibited proliferation and promoted apoptosis, increased ROS levels and caused DNA damage in transplanted cells. Taken together, the findings of the present study confirmed that PQQ induced apoptosis in human chondrosarcoma SW1353 cells and transplanted cells, by increasing intracellular ROS and reducing the ability of scavenging oxygen free radicals.
\end{abstract}

\section{Introduction}

Chondrosarcoma is the second most commonly occurring primary bone malignancy, which affects the pelvis, long bones and the spine, in addition to the larynx, head and neck, and

Correspondence to: Dr Chuanwen You, Department of Oncology, Suqian People's Hospital of Nanjing Drum Tower Hospital Group, 138 Huanghe South Road, Suqian, Jiangsu 223800, P.R. China E-mail: youchuanwen1321@163.com

Abbreviations: $\mathrm{PQQ}$, pyrroloquinoline quinone; ROS, reactive oxygen species; $\mathrm{O}_{2}^{-}$, superoxide; $\mathrm{OH}$, hydroxyl radical; TBS/T, Tris-buffered saline/ $0.1 \%$ Tween-20

Key words: chondrosarcoma, pyrroloquinoline quinone, reactive oxygen species, apoptosis it eventually metastasizes (1-3). Currently, chondrosarcoma remains largely incurable due to poor prognosis and a high rate of recurrence (4). Thus, the development of innovative therapies for this disease is required.

Pyrroloquinoline quinone (PQQ) is a nutrient widely distributed in nature and serves as a noncovalently bound redox cofactor in a series of bacterial quinoprotein dehydrogenases (5). PQQ scavenges reactive oxygen species (ROS) in bacteria (6). Previous studies have demonstrated that PQQ protected isolated liver mitochondria from damage following oxidative stress (7) and scavenged superoxide radicals $(8,9)$. A previous study revealed that PQQ could induce apoptosis in human promonocytic leukemia U937 and lymphoma EL-4 cells, in addition to Jurkat cell programmed death (7). The underlying mechanism may be relevant to the increase in intracellular ROS and the depletion of glutathione (GSH) (10). In addition, PQQ may induce tumor cell (A549, Neuro-2A and HCC-LM3) apoptosis by decreasing the expression of B-cell lymphoma 2 (11).

Oxidative stress arises from an imbalanced redox status between the production of ROS and the biological system able to remove them. ROS, including superoxide $\left(\mathrm{O}_{2}{ }^{-}\right)$, hydroxyl radical $(\bullet \mathrm{OH})$ and $\mathrm{H}_{2} \mathrm{O}_{2}$, are constantly generated in aerobic organisms (12). ROS can cause fatal lesions in cells under oxidative stress, leading to a number of diseases including cancer (13). High levels of ROS are detrimental and induce cell apoptosis or necrosis $(14,15)$. Recently, 'oxidation therapy' has been developed by inducing cytotoxic oxidative stress for cancer treatment. A number of antitumor agents, including vinblastine, cisplatin, doxorubicin, camptothecin and several others have exhibited antitumor activity via the ROS-dependent activation of apoptotic cell death, suggesting the potential use of ROS as an antitumor agent (16). However, the mechanisms underlying the role of PQQ in regulating chondrosarcoma cells have not been fully elucidated.

The present study examined the role of PQQ in chondrosarcoma cells, and identified that PQQ could increase cell apoptosis and the level of ROS. PQQ reduced the ability of scavenging oxygen free radicals by inhibiting the activation of superoxide dismutase (SOD)1 and SOD2, and the formation of GSH, causing an increased level of ROS. Additionally, an animal model was established in vivo, which identified that PQQ inhibited the proliferation of transplanted tumor cells, 
increased cell apoptosis through ROS and increased DNA damage. Thus, PQQ may be a desirable drug for cancer treatment in the future.

\section{Materials and methods}

Cell culture and reagents. The chondrosarcoma SW1353 cells, osteosarcoma Saos-2 cells and 293 cells were purchased from American Type Culture Collection (Manassas, VA, USA). Human XJH B lymphocytes were purchased from the Institute of Biochemistry and Cell Biology, the Chinese Academy of Sciences (Shanghai, China). All cells were cultured in Dulbecco's modified Eagle's medium (DMEM; Gibco; Thermo Fisher Scientific, Inc., Waltham, MA, USA) supplemented with $10 \%$ fetal bovine serum (FBS; Gibco; Thermo Fisher Scientific, Inc.) and $1 \%$ penicillin/streptomycin (Sigma-Aldrich; Merck KGaA, Darmstadt, Germany). All cells were maintained at $37^{\circ} \mathrm{C}$ with $5 \% \mathrm{CO}_{2}$ in an incubator with a constant humidity. PQQ was purchased from Sigma-Aldrich (Merck KGaA).

Cell cytotoxicity death analysis. Cell cytotoxic death was assessed using a CytoTox-Glo ${ }^{\mathrm{TM}}$ Cytotoxicity assay (Promega Corporation, Madison, WA, USA) according to the manufacturer's protocols. Briefly, all of the cells were seeded in at a density of $1 \times 10^{4}$ cells per well in $3 \mathrm{ml}$ culture medium and incubated at $37^{\circ} \mathrm{C}$ in $5 \% \mathrm{CO}_{2}$ for $6 \mathrm{~h}$. All of cells were incubated in culture medium with indicated concentration of PQQ $(0,40,80,120$ and $200 \mu \mathrm{M})$ for $24 \mathrm{~h}$ or $120 \mu \mathrm{M}$ PQQ on SW1353 cells at different time points $(6,12,24,36$ and $48 \mathrm{~h})$. A total of $50 \mu \mathrm{l}$ CytoTox-Glo ${ }^{\text {TM }}$ Reagent was first added and incubated at room temperature for $15 \mathrm{~min}$, luminescence was measured to determine dead cell luminescence. Then lysis reagent was added and luminescence was measured to determine total cell luminescence after $15 \mathrm{~min}$ of incubation at room temperature. The luminescent signal was adjusted to reflect the 'live cell' contribution by subtracting the initial dead cell signal.

Western blot analysis. The SW1353 cells were lysed with cell lysis buffer 10X (cat. no. 9803; Cell Signaling Technology, Inc., Danvers, MA, USA), which contained protease inhibitors (Sigma-Aldrich; Merck KGaA). Then cells were centrifuged at $12,000 \mathrm{x}$ g for $5 \mathrm{~min}$ at $4^{\circ} \mathrm{C}$. The supernatant was collected and a bicinchoninic acid Protein Assay kit (Sigma-Aldrich; Merck $\mathrm{KGaA}$ ) was used to measure the protein concentration. The protein samples $(20 \mu \mathrm{g} /$ lane $)$ were separated by $10 \%$ SDS-PAGE and transferred onto polyvinylidene difluoride membranes (EMD Millipore, Billerica, MA, USA). Membranes were blocked with Tris-buffered saline (TBS) and $0.1 \%$ Tween-20 (TBS/T) containing 5\% bovine serum albumin (Sangon Biotech Co., Ltd., Shanghai, China) at $37^{\circ} \mathrm{C}$ for $2 \mathrm{~h}$, and incubated overnight at $4^{\circ} \mathrm{C}$ with primary antibodies (Abcam, Cambridge, MA, USA; SOD1 and SOD2; 1:1,000 in TBS/T; SOD1; cat. no. ab13498; SOD2, cat. no. ab13534). The membrane was washed 3 times with TBS/T and then incubated with a horseradish peroxidase (HRP)-labeled secondary antibody (Cell Signaling Technology, Inc.; 1:2,000 in TBS/T; cat no. 7074) for $2 \mathrm{~h}$ at room temperature. The protein bands were detected by chemiluminescence (GE Healthcare,
Piscataway, NJ, USA). Bands were quantified by densitometry using Image Lab 5.0 software (Bio-Rad Laboratories, Inc., Hercules, CA, USA) and $\beta$-actin (Cell Signaling Technology, Inc.; 1:2,000; cat no. 4970) was used as an internal control.

Cell apoptosis analysis. SW1353 and Saos-2 cells were seeded into 6 -well plates at a density of $2 \times 10^{5}$ cells/well with the complete medium and grown for $24 \mathrm{~h}$. Following incubation, cells were exposed to $120 \mu \mathrm{M}$ PQQ or $200 \mu \mathrm{l}$ PBS (control group) at $37^{\circ} \mathrm{C}$ in a $5 \% \mathrm{CO}_{2}$ humidified atmosphere. Following treatment for $48 \mathrm{~h}$, cells were processed with trypsin EDTA $(0.25 \%)$ solution and centrifuged at $1,000 \times \mathrm{g}$ for $5 \mathrm{~min}$ at $4^{\circ} \mathrm{C}$. Following washing twice with PBS, $10 \mu 1$ Annexin $\mathrm{V}$-fluorescein isothiocyanate and $5 \mu \mathrm{l}$ propidium iodide (BD Biosciences, Franklin Lakes, NJ, USA) were added and incubated for $15 \mathrm{~min}$ in the dark at room temperature. Subsequently, apoptotic cells were detected by flow cytometry using a FACSC alibur system with Cell Quest software version 5.1 (BD Biosciences).

Intracellular ROS measurement. 2',7'-Dichlorodihydrofluorescein diacetate (DCFH-DA) was used as a probe for cellular ROS in the present study. SW1353 cells $\left(2 \times 10^{5} /\right.$ well $)$ in a 6 -well plate were incubated with PQQ $(120 \mu \mathrm{M})$ for $24 \mathrm{~h}$. Following incubation in serum-free medium containing $20 \mu \mathrm{M}$ DCFH-DA for $20 \mathrm{~min}$ at $37^{\circ} \mathrm{C}$, cells in each well were washed three times with PBS, digested by pancreatic enzymes at $37^{\circ} \mathrm{C}$ for $3 \mathrm{~min}$ and immediately subjected to ROS measurement by flow cytometry analysis using Cytomics FC $500 \mathrm{MCL}$ (Beckman Coulter, Inc., Brea, CA, USA). The wavelength was $488 \mathrm{~nm}$ for excitation and $525 \mathrm{~nm}$ for emission.

Hydroxyl radical $(\bullet \mathrm{OH})$ measurement. The deoxyribose degradation method was used to detect the level of $\bullet \mathrm{OH}$ as described by Baliga (17). Briefly, 2-deoxy-d-ribose at $3 \mathrm{mM}$ was added to cells just prior to the addition of PQQ. After incubation, $0.5 \mathrm{ml}$ of medium was collected and mixed with $0.5 \mathrm{ml}$ of $1 \%(\mathrm{w} / \mathrm{v})$ 2-thiobarbituric acid in $50 \mathrm{mM} \mathrm{NaOH}$ and $0.5 \mathrm{ml}$ of $2.8 \%(\mathrm{w} / \mathrm{v})$ trichloroacetic acid. The mixture was then heated at $100^{\circ} \mathrm{C}$ for $15 \mathrm{~min}$, cooled, and extracted with n-butanol. The supernatant was measured for absorbance at $532 \mathrm{~nm}$.

GSH production. A GSH Assay kit (Beyotime Institute of Biotechnology, Shanghai, China) was used to assess the content of GSH according to the manufacturer's protocols (18).

Tumor xenograft experiments. Experimental procedures were conducted in conformity with institutional guidelines (The Second Affiliated Hospital of Zhejiang University School of Medicine, Zhejiang, China) for the care and use of laboratory animals, and ethical approval was obtained from The Second Affiliated Hospital of Zhejiang University School of Medicine. Procedures also conformed to the National Institutes of Health Guide for Care and Use of Laboratory Animals (19). A mouse model of chondrosarcoma was established using SW1353 cells to generate subcutaneous xenografts. A total of 12 Male BALB/c nude mice (6 week-old, 18-22 g) were obtained from the Shanghai Laboratory Animal Center, Chinese Academy Sciences (Shanghai, China), and housed in a controlled 12-h 
light/dark cycle environment at $20-25^{\circ} \mathrm{C}$ with free access to food and water. The mice were randomly divided into 2 groups (n=6/group). Briefly, $\sim 1 \times 10^{6}$ cells/mouse were injected subcutaneously once into the lateral flanks of BALB/c nude mice and 1 tumor per mouse was grown over 10-14 days until they almost reached $100-150 \mathrm{~mm}^{3}$. The mice were then intraperitoneally injected with $50 \mathrm{mg} / \mathrm{kg}$ PQQ or $200 \mu \mathrm{l}$ PBS once daily for 10 days. Following 10 days, the mice were sacrificed and the tumor xenografts were harvested. The tumor volume was measured and calculated as follows: Volume $=\left(\right.$ width $^{2} \mathrm{x}$ length) $/ 2$. The tumor tissues were stored in $-80^{\circ} \mathrm{C}$.

Immunohistochemistry. Sections $(4-\mu \mathrm{m})$ of tumor tissues which were cut using a LEICA RM2235 (Leica Microsystems $\mathrm{GmbH}$, Wetzlar, Germany) and then were fixed in a $10 \%$ formalin solution (Sigma-Aldrich; Merck KGaA) for $24 \mathrm{~h}$ at room temperature, embedded in paraffin wax (Sigma-Aldrich; Merck KGaA). Then they were deparaffinized with xylene for 10 min twice, 100\% ethyl alcohol (Sangon Biotech Co., Ltd., Shanghai, China) for 5 min twice, 95\% ethyl alcohol for $5 \mathrm{~min}, 85 \%$ ethyl alcohol for $5 \mathrm{~min}, 75 \%$ ethyl alcohol for $5 \mathrm{~min}$, PBS washed three times. Next, the endogenous peroxidase activity was quenched using $3 \% \mathrm{H}_{2} \mathrm{O}_{2}$ and nonspecific binding was blocked with 5\% FBS at room temperature for $20 \mathrm{~min}$. Following incubation with primary antibody proliferating cell nuclear antigen (PCNA) and index $\gamma-\mathrm{H} 2 \mathrm{~A}$ histone member X ( $\gamma$-H2AX; Abcam, Cambridge, MA, USA; PCNA cat. no. ab18197, $\gamma$-H2AX; cat. no. ab2893; all diluted 1:1,000 in TBS/T) overnight at $4^{\circ} \mathrm{C}$, the sections were further incubated with the corresponding HRP-conjugated secondary antibody (Cell Signaling Technology, Inc.; 1:2,000 in TBS/T; cat. no. 7074) for $1 \mathrm{~h}$ at room temperature. The target protein expression was visualized using 3,3'-diaminobenzidine and hematoxylin counterstaining was also performed at room temperature for $3 \mathrm{~min}$. The negative control was established using PBS rather than the primary antibody. The sections were observed under light microscopy (Olympus Corporation, Tokyo, Japan). The positive rates were measured using Image-Pro Plus v 6.0 software (Media Cybernetics, Inc., Rockville, MD, USA).

In situ quantification of apoptotic cells. Apoptotic cells were detected using terminal deoxynucleotidyl transferase dUTP nick end labeling (TUNEL) staining and an in situ cell death detection kit (Roche Diagnostics, Basel, Switzerland) according to the manufacturer's protocols. Stained sections were visualized under a fluorescence microscope. TUNEL-positive cells with brown staining and all cells with nuclear hematoxylin counterstaining for $30 \mathrm{sec}$ at room temperature were counted within 5 randomly selected fields under high-power magnification (DM-2,500; Leica Microsystems GmbH). The index of apoptosis was expressed as the ratio of positively stained apoptotic cells to the total number of cells counted, $\mathrm{x} 100 \%$.

Statistical analysis. Data were expressed as the mean \pm standard deviation $(\mathrm{n}=3)$ and analysis was performed using Prism 5 software (Graph Pad Software, Inc., La Jolla, CA, USA). UCSF DOCK 6.5 Software (University of California, San Francisco, CA, USA) was used to analyze the molecular docking. Statistical differences between two groups were examined with the Student's t-test and multiple groups were compared using one-way analysis of variance followed by Tukey's post hoc test. $\mathrm{P}<0.05$ was considered to indicate a statistically significant difference.

\section{Results}

$P Q Q$ enhances the apoptotic rate in chondrosarcoma $S W 1353$ cells. To determine whether PQQ has a role in causing chondrosarcoma cell death, a cell cytotoxicity assay was performed to measure cell viability. SW1353, Saos-2, 293 and XJH B cells were treated with different concentrations of PQQ $(0$, $40,80,120$ and $200 \mu \mathrm{M}$ ) for $24 \mathrm{~h}$. The results demonstrated that chondrosarcoma SW1353 cells and osteosarcoma Saos-2 cells had a greater percentage of apoptosis than normal human 293 and XJH B cells, which increased in a PQQ concentration-dependent manner (Fig. 1A). It was identified that $120 \mu \mathrm{M}$ PQQ treatment significantly increased the cell death rate in a time-dependent manner as measured by a cell cytotoxicity analysis (Fig. 1B). Flow cytometry was used to measure the apoptotic rates of chondrosarcoma SW1353 cells. It was identified that treatment with PQQ markedly increased the number of apoptotic SW1353 and Saos-2 cells when compared with the PBS treatment groups (Fig. 1C and D). These results indicated that cell death was increased in a dose- and time-dependent manner, while the effect on normal cells was relatively small.

$P Q Q$ enhances the level of ROS. To determine whether PQQ-induced cell apoptosis was associated with oxidative stress levels in chondrosarcoma SW1353 cells, DCFH-DA staining and flow cytometry were used to detect the changes in the levels of ROS and hydroxyl radicals in SW1353 cells treated with $120 \mu \mathrm{M}$ PQQ for $24 \mathrm{~h}$. It was identified that the level of ROS and hydroxyl radicals increased when compared with the control PBS group ( $\mathrm{P}<0.05$ and $\mathrm{P}<0.01$; Fig. 2). These results demonstrated that $\mathrm{PQQ}$ treatment increased oxidative stress levels in chondrosarcoma SW1353 cells.

$P Q Q$ inhibits the activity of SOD1 and SOD2, and reduces GSH production. To investigate the molecular mechanism of PQQ-induced oxidative stress in chondrosarcoma SW1353 cells, western blotting was performed to detect the total SOD activity and to examine the expression of SOD1 and SOD2 protein. The results indicated that, when compared with control, the total SOD activity was reduced when cells were treated with different concentration of PQQ $(0,40,80$ and $120 \mu \mathrm{M}$ ) PQQ for $24 \mathrm{~h}$ (Fig. 3A); however, no significant difference was observed in SOD1 and SOD2 protein expression (Fig. 3B). Molecular docking software UCSF DOCK 6.5 (University of California) was used to test molecular docking simulations of PQQ with SOD1 and SOD2 protein, respectively. It was observed that PQQ formed 3 hydrogen bonds with the amino acids that surround the SOD1 activity center (Fig. 3C; Table I). Similarly, PQQ formed 4 hydrogen bonds with the amino acids that surround the SOD1 activity center (Fig. 3D; Table II). The content of GSH was also detected, and it was identified that GSH content was significantly decreased following treatment with different concentrations of PQQ $(0,40,80$ and $120 \mu \mathrm{M})$ for $24 \mathrm{~h}$ (Fig. 3E). These results indicated that PQQ can decrease the level of ROS by inhibiting 


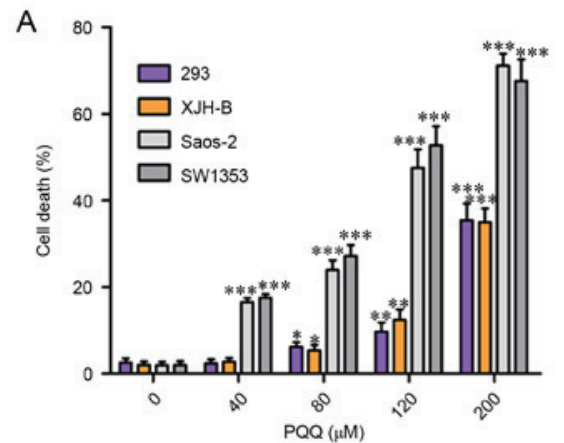

B
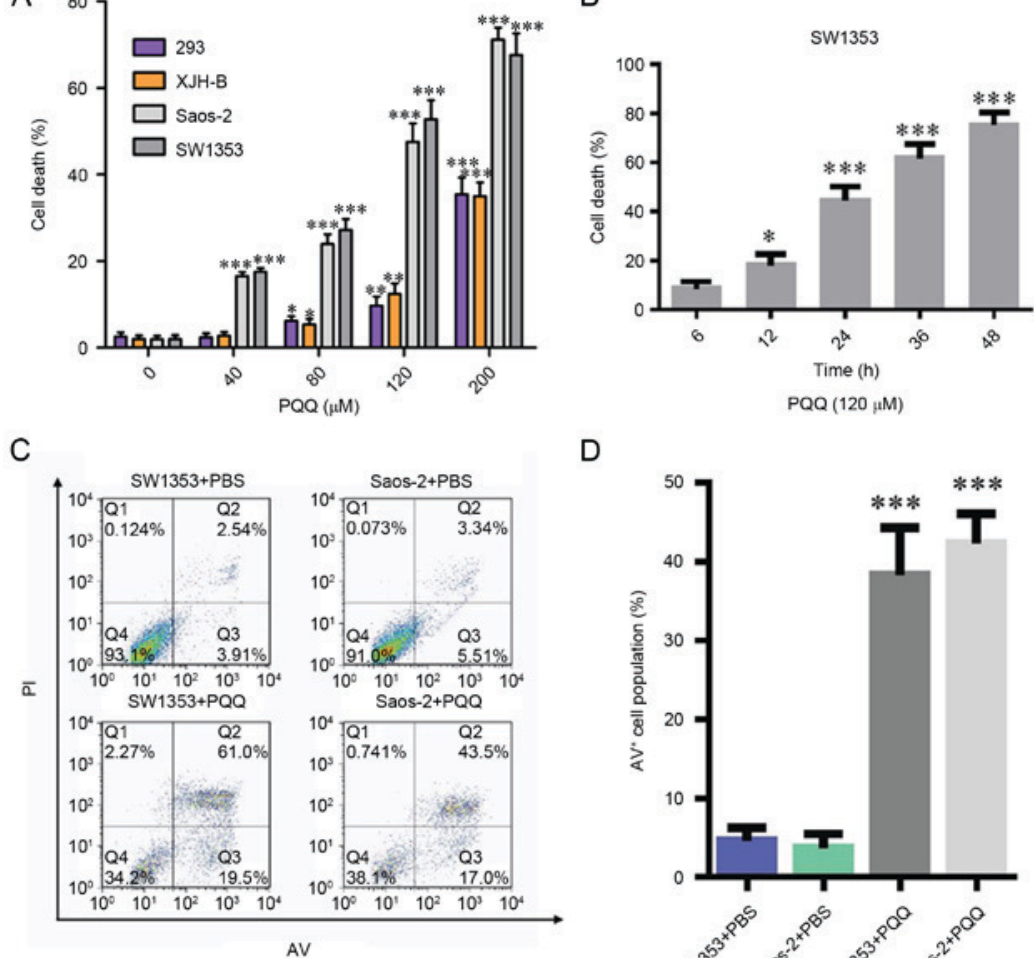

D

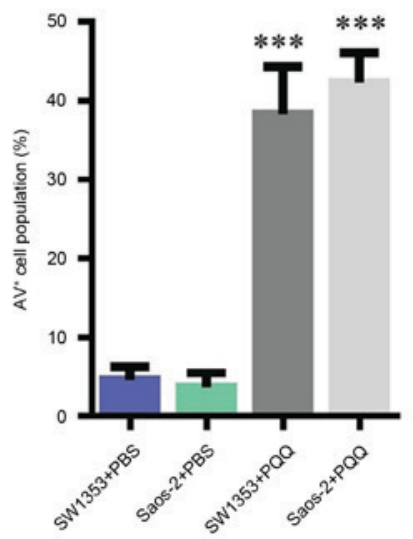

Figure 1. (A) Different PQQ sensitivity in SW1353, Saos-2, 293 and XJH B cells. All cells were incubated with PQQ (0, 40, 80, 120 or $200 \mu \mathrm{M})$ for 48 h. Cell viability was measured using cell cytotoxicity assay. ${ }^{*} \mathrm{P}<0.05,{ }^{* *} \mathrm{P}<0.01,{ }^{* * *} \mathrm{P}<0.001$ vs. PQQ $(0 \mu \mathrm{M})$. (B) Effects of PQQ $(120 \mu \mathrm{M})$ on SW1353 cells at different time points $(6,12,24,36$ and $48 \mathrm{~h}) .{ }^{*} \mathrm{P}<0.05$ and ${ }^{* * *} \mathrm{P}<0.001$ vs. $6 \mathrm{~h}$. (C) The number of apoptotic cells was determined by flow cytometry in SW1353 and Saos-2 cells following treatment with $\mathrm{PQQ}$ for $24 \mathrm{~h}$. (D) The rate of apoptosis represented as a histogram. ${ }^{* * *} \mathrm{P}<0.001$ vs. PBS treatment. PQQ, pyrroloquinoline quinone; PI, propidium iodide; AV, Annexin V-fluorescein isothiocyanate.
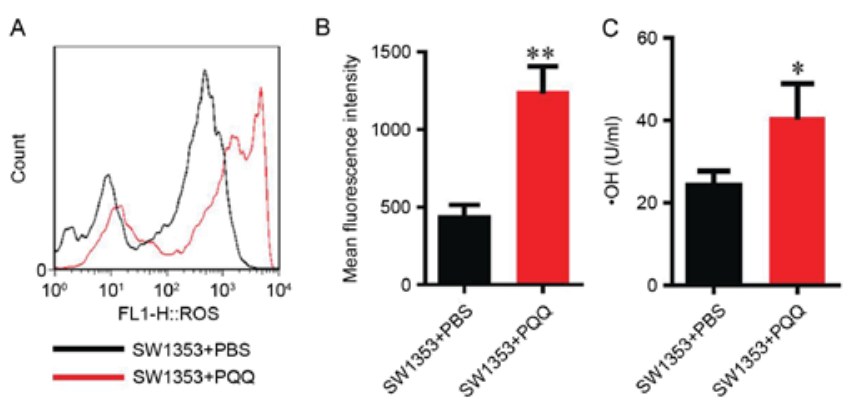

Figure 2. (A) Flow cytometry was used to detect ROS levels following treatment with or without PQQ for $24 \mathrm{~h}$. (B) Histogram of the levels of ROS in SW1353 cells treated with $120 \mu \mathrm{M}$ PQQ for 24 h. (C) Deoxyribose degradation method was used to determine the levels of $\mathrm{OH}$ in SW1353 cells treated with $120 \mu \mathrm{M}$ PQQ for $24 \mathrm{~h} .{ }^{*} \mathrm{P}<0.05$ and ${ }^{* *} \mathrm{P}<0.01$ vs. PBS treatment. ROS, reactive oxygen species; $\mathrm{PQQ}$, pyrroloquinoline quinone; $\mathrm{OH}$, hydroxyl radicals.

the activities of SOD1 and SOD2, and the production of GSH, reducing the ability of scavenging oxygen free radicals in chondrosarcoma SW1353 cells.

$P Q Q$ increases the inhibitory effect on tumorigenesis in vivo. To clarify the effect of PQQ in vivo, SW1353 cells were xenografted into BALB/c nude mice. When compared with the PBS control, the tumor volume of the PQQ treated group was significantly smaller (Fig. 4A and B). Immunohistochemistry analysis demonstrated that the percentage of PCNA positive cells was decreased and in TUNEL analysis the number of

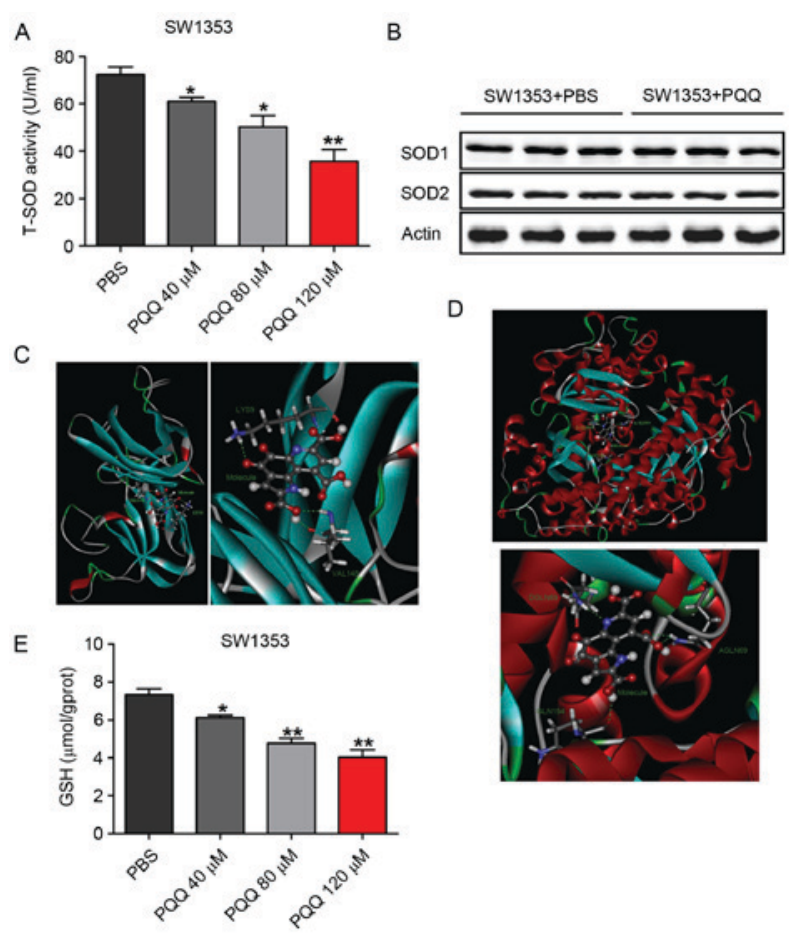

Figure 3. Western blot analysis was performed to analyze the protein levels of (A) total SOD, and (B) SOD1 and SOD2 in SW1353 cells. (C) A schematic diagram of PQQ combined with SOD1. (D) Schematic diagrams of PQQ combined with SOD2. (E) The level of GSH in chondrosarcoma SW1353 cells treated with or without PQQ. ${ }^{*} \mathrm{P}<0.05$ and ${ }^{* *} \mathrm{P}<0.01$ vs. $\mathrm{PBS}$. SOD, superoxide dismutase; T-SOD, total SOD; $\mathrm{PQQ}$, pyrroloquinoline quinone; GSH, glutathione. 
Table I. Pyrroloquinoline quinone combined with superoxide dismutase 1 hydrogen bond.

\begin{tabular}{lcccc}
\hline $\mathrm{X}-\mathrm{H} \ldots \mathrm{Y}$ & $d_{(\mathrm{X}-\mathrm{H})}(\mathrm{A})$ & $d_{(\mathrm{H} \ldots \mathrm{Y})}(\mathrm{A})$ & $d_{(\mathrm{X} \ldots \mathrm{Y})}(\mathrm{A})$ & $\angle \mathrm{XHY}\left({ }^{\circ}\right)$ \\
\hline Molecule: $\mathrm{O}_{13} \ldots \mathrm{H}_{22}:$ LYS9 & 1.04 & 1.90 & 2.69 & 130 \\
Molecule: $\mathrm{O}_{12} \ldots \mathrm{H}_{\mathrm{N}}$ : VAL148 & 1.00 & 2.06 & 2.79 & 128 \\
Molecule: $\mathrm{H}_{27} \ldots \mathrm{O}$ : VAL148 & 0.95 & 1.97 & 2.76 & 139
\end{tabular}

Table II. Pyrroloquinoline quinone combined with superoxide dismutase 2 hydrogen bond.

\begin{tabular}{|c|c|c|c|c|}
\hline $\mathrm{X}-\mathrm{H} \ldots \mathrm{Y}$ & $d_{(\mathrm{X}-\mathrm{H})}(\mathrm{A})$ & $d_{(\mathrm{H} \ldots \mathrm{Y})}(\mathrm{A})$ & $d_{(\mathrm{X} \ldots \mathrm{Y})}(\mathrm{A})$ & $\angle X H Y\left({ }^{\circ}\right)$ \\
\hline Molecule: $\mathrm{O}_{20} \ldots \mathrm{HE}_{21}$ :GLN69 & 1.00 & 2.02 & 2.77 & 129 \\
\hline Molecule: $\mathrm{O}_{21} \ldots \mathrm{HE}_{21}:$ GLN69 & 1.00 & 2.28 & 3.06 & 134 \\
\hline Molecule: $\mathrm{N}_{16} \ldots \mathrm{HE}_{22}: \mathrm{GLN} 69: \mathrm{D}$ & 1.00 & 2.11 & 3.10 & 170 \\
\hline Molecule: $\mathrm{H}_{27} \ldots \mathrm{O}: \mathrm{GLN} 154$ & 0.95 & 2.13 & 2.81 & 127 \\
\hline
\end{tabular}

A

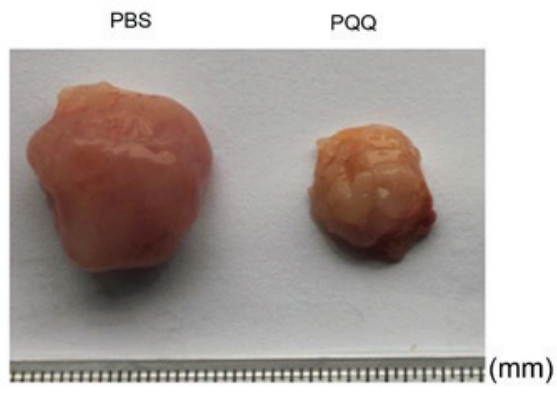

C

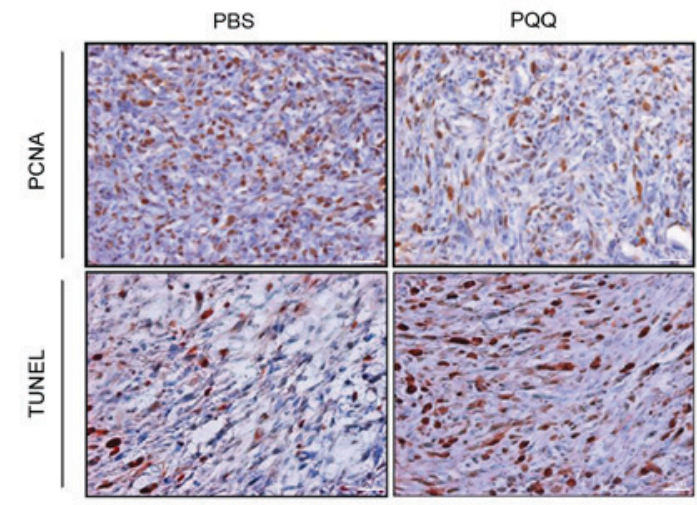

B
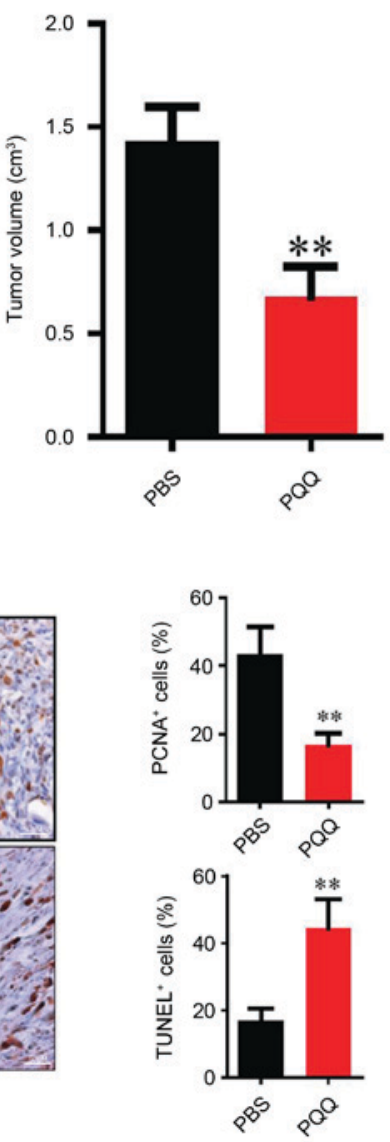

Figure 4. (A) Compared with the PBS group, treatment with PQQ delayed tumor growth and decreased tumor size. (B) The tumor volume of those treated with PQQ was reduced when compared with the PBS group. (C) PCNA was used to detect the proliferation of tumor cells. Scale bar $50 \mu \mathrm{M}$. (D) Immunostaining with TUNEL was used to examine cell apoptosis. ${ }^{* *} \mathrm{P}<0.01$ vs. PBS. PQQ, pyrroloquinoline quinone; PCNA, proliferating cell nuclear antigen; TUNEL, terminal deoxynucleotidyl transferase dUTP nick end labeling.

apoptotic cells increased when compared with the PBS control (Fig. 4C and D). These findings indicated that PQQ may inhibit the proliferation and promote the apoptosis of tumor cells.

$P Q Q$ increases the level of ROS and DNA damage in tumor xenograft cells. To clarify whether PQQ decreased the proliferation of tumor xenograft cells and if increased apoptosis was associated with ROS, the ROS and DNA damage associated index $\gamma$-H2AX was detected in the tumors. The results demonstrated that the levels of ROS in the PQQ treated group were significantly higher compared with the PBS control group (Fig. 5A and B), and the percentage of $\gamma$-H2AX 
A

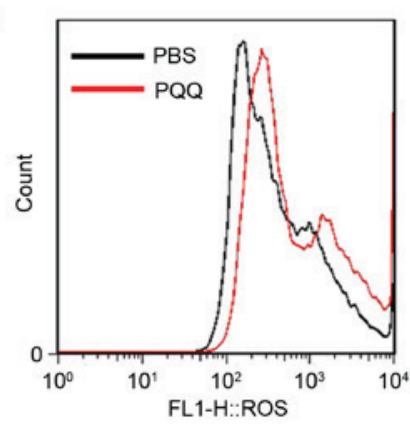

B

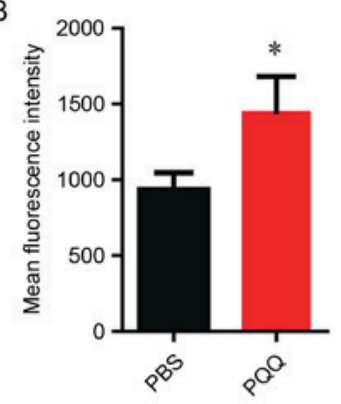

C
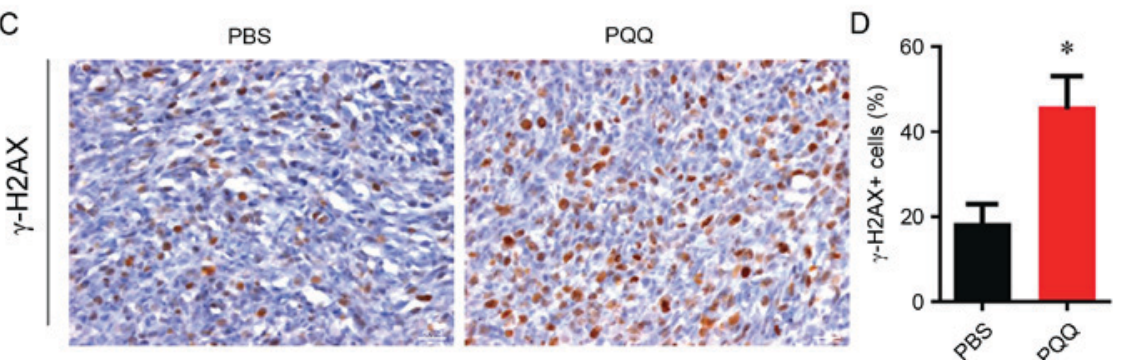

Figure 5. (A) Flow cytometry was used to detect (B) ROS levels in tumor xenograft cells. (C) Immunohistochemical staining was used to detect $\gamma$-H2AX and (D) the histogram presents the percentage of $\gamma$-H2AX positive cells. ${ }^{*} \mathrm{P}<0.05$ vs. PBS. PQQ, pyrroloquinoline quinone; ROS, reactive oxygen species; $\gamma$-H2AX, $\gamma$-H2A histone member X.

was significantly increased (Fig. 5C and D). These results suggested that PQQ increased intracellular ROS and caused DNA damage in transplanted cells.

\section{Discussion}

Chondrosarcoma is a malignant tumor of mesenchymal origin that is generally locally aggressive and tends to produce early systemic metastases. However, chondrosarcoma does not respond to chemotherapy or radiation. Therefore, novel therapies are required. PQQ was first identified in bacteria and is also likely to be important in mammals (20-22). Previous studies on PQQ have been focused on its activities as an antioxidant and redox modulator $(7)$, a cardio- and neuro-protectant $(23,24)$, and its radio-protective effects on the hemopoietic system (25). PQQ has a potent antitumor effect and possesses a significant cytotoxic effect on human lung adenocarcinoma, hepatocarcinoma, melanoma cells and brain cancer; however, it exhibits little effect on normal cells, which suggests that PQQ may be an effective therapeutic drug in the future $(7,11,26)$. However, the molecular mechanism of PQQ underlying its effect on chondrosarcoma remains to be elucidated.

The present study examined cell death following treatment with or without different concentrations of PQQ in cancer and normal cells. It was identified that cell death increased in a dose- and time-dependent manner following treatment with PQQ, while the effect on normal cells was relatively small. Cell death occurs in a variety of ways, including apoptosis, necrosis and cell autophagy $(27,28)$; however, the most frequently studied and most common type is apoptosis. In the present study it was hypothesized whether the effect of PQQ on chondrosarcoma cells was achieved by promoting the rate of apoptosis in chondrosarcoma cells. In the present study, the apoptotic level following PQQ treatment in SW1353 and Saos-2 cells was investigated by flow cytometry. The results demonstrated a large level of apoptosis occurred following PQQ treatment, and the proportion of cell apoptosis was comparable to that of the cytotoxic cell death rate, indicating that PQQ may mainly induce apoptosis to kill chondrosarcoma cells. Previous studies have reported that PQQ may cause apoptosis by inhibiting the synthesis of $\mathrm{GSH}$ and producing $\mathrm{H}_{2} \mathrm{O}_{2}$ via autoxidation to increase the level of oxidative stress in tumor cells $(7,11)$. It is well known that ROS induces cell death, including apoptosis. In addition, the enhancement of ROS production has long been associated with the apoptotic response induced by several anticancer agents (29-31). The status of intracellular redox is regulated by antioxidant enzymes (including SOD, catalase and GSH peroxidase) and non-enzymatic antioxidants (such as GSH and vitamin C) (11). The levels of ROS and hydroxyl radicals in the PQQ treated and the PBS control groups were compared using flow cytometry and deoxyribose degradation method. The results suggested that PQQ can cause a significant increase in oxidative stress levels in the SW1353 chondrosarcoma cell line.

It was demonstrated that treatment with PQQ had inhibitory effects on tumorigenesis and decreased tumor size when compared with the control in vivo, which is similar to the results of the in vitro studies. However, it is unclear whether the mechanism of PQQ inhibition in the xenograft tumors is consistent with the in vitro results. These findings indicated that the levels of ROS in the PQQ treated group were significantly higher than those in the PBS control group, and the percentage of $\gamma-\mathrm{H} 2 \mathrm{AX}$ positive cells in the DNA injury-associated indexes was significantly increased. The results suggested that PQQ may inhibit the proliferation of tumor xenograft cells by promoting oxidative stress and increasing the percentage of $\gamma-\mathrm{H} 2 \mathrm{AX}$. In conclusion, the mechanism underlying PQQ-induced apoptosis may be associated with increasing the levels of ROS, suggesting 
that PQQ may be a potential drug for chondrosarcoma therapies.

\section{References}

1. Bauer HC, Brosjö O, Kreicbergs A and Lindholm J: Low risk of recurrence of enchondroma and low-grade chondrosarcoma in extremities. 80 patients followed for 2-25 years. Acta Orthop Scand 66: 283-288, 1995.

2. Eriksson AI, Schiller A and Mankin HJ: The management of chondrosarcoma of bone. Clin Orthop Relat Res: 44-66, 1980.

3. Bovée JV, Cleton-Jansen AM, Taminiau AH and Hogendoorn PC: Emerging pathways in the development of chondrosarcoma of bone and implications for targeted treatment. Lancet Oncol 6: 599-607, 2005

4. Bao X, Ren T, Huang Y, Wang S, Zhang F, Liu K, Zheng B and Guo W: Induction of the mesenchymal to epithelial transition by demethylation-activated microRNA-125b is involved in the anti-migration/invasion effects of arsenic trioxide on human chondrosarcoma. J Exp Clin Cancer Res 35: 129, 2016.

5. McIntire WS: Newly discovered redox cofactors: Possible nutritional, medical, and pharmacological relevance to higher animals. Annu Rev Nutr 18: 145-177, 1998.

6. Misra HS, Khairnar NP, Barik A, Indira Priyadarsini K, Mohan H and Apte SK: Pyrroloquinoline-quinone: A reactive oxygen species scavenger in bacteria. FEBS Lett 578: 26-30, 2004.

7. He K, Nukada H, Urakami T and Murphy MP: Antioxidant and pro-oxidant properties of pyrroloquinoline quinone (PQQ): Implications for its function in biological systems. Biochem Pharmacol 65: 67-74, 2003.

8. Smidt CR, Steinberg FM and Rucker RB: Physiologic importance of pyrroloquinoline quinone. Proc Soc Exp Biol Med 197: 19-26, 1991.

9. Bishop A, Gallop PM and Karnovsky ML: Pyrroloquinoline quinone: A novel vitamin? Nutr Rev 56: 287-293, 1998.

10. Shankar BS, Pandey R, Amin P, Misra HS and Sainis KB: Role of glutathione in augmenting the anticancer activity of pyrroloquinoline quinone (PQQ). Redox Rep 15: 146-154, 2010.

11. Min Z, Wang L, Jin J, Wang X, Zhu B, Chen H and Cheng Y: Pyrroloquinoline quinone induces cancer cell apoptosis via mitochondrial-dependent pathway and down-regulating cellular Bcl-2 protein expression. J Cancer 5: 609-624, 2014.

12. Pizzimenti S, Toaldo C, Pettazzoni P, Dianzani MU and Barrera G: The'two-faced' effects of reactive oxygen species and the lipid peroxidation product4-hydroxynonenal in the hallmarks of cancer. Cancers (Basel) 2: 338-363, 2010.

13. Tripathi DN, Chowdhury R, Trudel LJ, Tee AR, Slack RS, Walker CL and Wogan GN: Reactive nitrogen species regulate autophagy through ATM-AMPK-TSC2 mediated suppression of mTORC1. Proc Natl Acad Sci USA 110: E2950-E2957, 2013.

14. Finkel T and Holbrook NJ: Oxidants, oxidative stress and the biology of ageing. Nature 408: 239-247, 2000.

15. Martindale JL and Holbrook NJ: Cellular response to oxidative stress: Signalling for suicide and survival. J Cell Physiol 192: $1-15,2002$.

16. Fang J, Nakamura $\mathrm{H}$ and Iyer AK: Regulation of replicative potential of cells. J Drug Target 15: 475-486, 2007.
17. Baliga R, Zhang Z, Baliga M, Ueda $\mathrm{N}$ and Shah SV: In vitro and in vivo evidence suggesting a role for iron in cisplatin-induced nephrotoxicity. Kidney Int 53: 394-401, 1998.

18. Xie X, Zhao Y, Ma CY, Xu XM, Zhang YQ, Wang CG, Jin J, Shen X, Gao JL, Li N, et al: Dimethyl fumarate induces necroptosis in colon cancer cells through GSH depletion/ROS increase/MAPKs activation pathway. Br J Pharmacol 172: 3929-3943, 2015

19. Wang X, Bao Y, Dong Z, Chen Q, Guo H, Ziang C and Shao J WP1130 attenuates cisplatin resistance by decreasing P53 expression in non-small cell lung carcinomas. Oncotarget 8: 49033-49043, 2017.

20. Salisbury SA, Forrest HS, Cruse WB and Kennard O: A novel coenzyme from bacterial primary alcohol dehydrogenases. Nature 280: 843-844, 1979

21. Killgore J, Smidt C, Duich L, Romero-Chapman N, Tinker D, Reiser K, Melko M, Hyde D and Rucker RB: Nutritional importance of pyrroloquinoline quinone. Science 245: 850-852, 1989.

22. Steinberg FM, Gershwin ME and Rucker RB: Dietary pyrroloquinoline quinone: Growth and immune response in BALB/c mice. J Nutr 124: 744-753, 1994.

23. Tao R, Karliner JS, Simonis U, Zheng J, Zhang J, Honbo N and Alano CC: Pyrroloquinoline quinone preserves mitochondrial function and prevents oxidative injury in adult rat cardiac myocytes. Biochem Biophys Res Commun 363: 257-262, 2007.

24. Hara $\mathrm{H}$, Hiramatsu $\mathrm{H}$ and Adachi T: Pyrroloquinoline quinone is a potentneuroprotective nutrient against 6-hydroxydopamine-induced neurotoxicity. Neurochem Res 32: 489-495, 2007.

25. Xiong XH, Zhao Y, Ge X, Yuan SJ, Wang JH, Zhi JJ, Yang YX, Du BH, Guo WJ, Wang SS, et al: Production and radioprotective effects of pyrroloquinoline quinone. Int J Mol Sci 12: 8913-8923, 2011.

26. Sato K and Toriyama M: Effect of pyrroloquinoline quinone (PQQ) on melanogenic protein expression in murine B16 melanoma. J Dermatol Sci 53: 140-145, 2009.

27. Fadeel B and Orrenius S: Apoptosis: A basic biological phenomenon with wide-ranging implications in human disease. J Intern Med 258: 479-517, 2005 .

28. Clarke PG: Developmental cell death: Morphological diversity and multiple mechanisms. Anat Embryol (Berl) 181: 195-213, 1990.

29. Tibodeau JD, Benson LM, Isham CR, Owen WG and Bible KC: The Anticanceragent chaetocin is a competitive substrate and inhibitor ofthioredoxin reductase. Antioxid Redox Signal 11: 1097-1106, 2009

30. Madan E, Prasad S, Roy P, George J and Shukla Y: Regulation of apoptosis by resveratrol through JAK/STAT and mitochondria mediated pathway in human epidermoid carcinoma A431 cells. Biochem Biophys Res Commun 377: 1232-1237, 2008.

31. Liu Z, Huang SL, Li MM, Huang ZS, Lee KS and Gu LQ Inhibition of thioredoxin reductase by mansonone $\mathrm{F}$ analogues: Implications for anticancer activity. Chem Biol Interact 177: 48-57, 2009.

This work is licensed under a Creative Commons Attribution-NonCommercial-NoDerivatives 4.0 International (CC BY-NC-ND 4.0) License. 